\title{
Binding-energy distribution and dephasing of localized biexcitons
}

\author{
Langbein, Wolfgang Werner; Hvam, Jørn Märcher; Umlauff, M.; Kalt, H.; Jobst, B.; Hommel, D.
}

Published in:

Physical Review B

Link to article, DOI:

10.1103/PhysRevB.55.R7383

Publication date:

1997

Document Version

Publisher's PDF, also known as Version of record

Link back to DTU Orbit

Citation $(A P A)$ :

Langbein, W. W., Hvam, J. M., Umlauff, M., Kalt, H., Jobst, B., \& Hommel, D. (1997). Binding-energy distribution and dephasing of localized biexcitons. Physical Review B, 55(12), R7383-R7386.

https://doi.org/10.1103/PhysRevB.55.R7383

\section{General rights}

Copyright and moral rights for the publications made accessible in the public portal are retained by the authors and/or other copyright owners and it is a condition of accessing publications that users recognise and abide by the legal requirements associated with these rights.

- Users may download and print one copy of any publication from the public portal for the purpose of private study or research.

- You may not further distribute the material or use it for any profit-making activity or commercial gain

- You may freely distribute the URL identifying the publication in the public portal 


\title{
Binding-energy distribution and dephasing of localized biexcitons
}

\author{
W. Langbein and J. M. Hvam \\ Mikroelektronik Centret, The Technical University of Denmark, Building 345 East, DK-2800 Lyngby, Denmark \\ M. Umlauff and H. Kalt \\ Institut für Angewandte Physik, Universität Karlsruhe, D-76128 Karlsruhe, Germany \\ B. Jobst and D. Hommel \\ Institut für Festkörperphysik, Universität Bremen, D-28334 Bremen, Germany
}

(Received 22 October 1996)

\begin{abstract}
We report on the binding energy and dephasing of localized biexciton states in narrow ZnSe multiple quantum wells. The measured binding-energy distribution of the localized biexcitons shows a width of $2.2 \mathrm{meV}$ centered at $8.5 \mathrm{meV}$, and is fairly independent of the exciton localization energy. In four-wave mixing, the biexciton photon echo decays fast and nonexponentially. This behavior results from the inhomogeneous broadening of the biexciton binding energy, as we show by a comparison with an analytical model calculation. The fast decay is thus not related to a fast microscopic biexciton dephasing. [S0163-1829(97)51912-6]
\end{abstract}

The formation and binding energy of biexcitons in semiconductor nanostructures has been investigated intensively since the availability of high-quality samples. It has been shown both theoretically ${ }^{1}$ and experimentally, ${ }^{2,3}$ that, by lowering the dimensionality, the biexciton binding energy is enhanced more strongly than the exciton binding energy. For two-dimensional systems, this leads to a biexciton binding energy of about $20 \%$ of the exciton binding energy, ${ }^{4,5}$ compared to $10 \%$ for three dimensions ${ }^{6}$ (Haynes rule). The formation of localized exciton states, present in real structures due to imperfections of the interfaces, ${ }^{7,8}$ leads to a further increase of the binding energy of the corresponding localized biexciton states. ${ }^{3,9}$ The different shapes of the exciton localization potentials should, in turn, give rise to an inhomogeneous broadening of the biexciton binding energy, even for a fixed energy of the localized excitons. To our knowledge, this has not been explicitly treated until now.

In this paper, we demonstrate that there is indeed a significant distribution of biexciton binding energies, and that this gives rise to a fast decay of the biexcitonic photon echo signal, which has previously been attributed to a fast microscopic dephasing of the biexciton. ${ }^{9,10}$ We study the distribution of biexciton binding energies in a $\mathrm{ZnSe}$ multiplequantum-well (MQW) sample, consisting of ten periods of 0.8-nm ZnSe wells and 20-nm $\mathrm{Zn}_{0.88} \mathrm{Mg}_{0.12} \mathrm{~S}_{0.18} \mathrm{Se}_{0.82}$ barriers, lattice matched to GaAs. It was grown by molecularbeam epitaxy (MBE) after a $200 \mathrm{~nm}$ GaAs buffer layer on a $\langle 001\rangle$ GaAs substrate. We use time-resolved photoluminescence (PL) and four-wave mixing (FWM) to investigate the the biexciton states localized by fluctuations in well width and barrier composition.

The nonresonantly excited, time-integrated PL of a sample piece with the GaAs substrate removed (Fig. 1) shows the dominant recombination of localized heavy-hole excitons $\left(X_{\mathrm{hh}}\right)$, a small amount of acceptor-bound excitons $\left(A_{0} X_{\mathrm{hh}}\right)$, and the $\mathrm{ZnSe}$ longitudinal-optical (LO)-phonon replica of the localized exciton PL $\left(X_{\mathrm{hh}}-\mathrm{LO}\right)$. The PL from localized excitons in the quaternary barriers $\left(X_{\text {barrier }}\right)$ is visible above the $X_{\mathrm{hh}}$ PL. The absorption spectrum (dotted) shows the $X_{\mathrm{hh}}$ exciton, the $X_{\text {barrier }}$ exciton and the onset of the barrier continuum $\left(C_{\text {barrier }}\right)$. The width of the $X_{\mathrm{hh}} \mathrm{PL}$ is $9 \mathrm{meV}$, and shows a Stokes shift of $7 \mathrm{meV}$ from the $X_{\mathrm{hh}}$ absorption, in reasonable agreement with the expected Stokes shift of $60 \%$ of the inhomogeneous linewidth in type-I QW's. ${ }^{11}$

The PL of the sample detected 100 ps after excitation (Fig. 2) reveals, at higher excitation intensities, a second transition below the $X_{\mathrm{hh}}$ PL. This is attributed to the recombination of localized biexcitons, in agreement with the linear scaling of the relative intensity of the biexciton $\left(B_{\mathrm{hh}}\right)$ and exciton $\left(X_{\mathrm{hh}}\right)$ PL with excitation intensity (inset of Fig. 2). Due to the strong inhomogeneous broadening of the exciton transition, only an average biexciton binding energy of 8 $\mathrm{meV}$ can be estimated, while the distribution is not accessible. The binding energy is comparable to reported values of 5-10 meV in similar MQW structures. ${ }^{12-16}$

We now discuss spectrally resolved FWM data obtained in reflection geometry using spectrally broad 100 fs or spec-

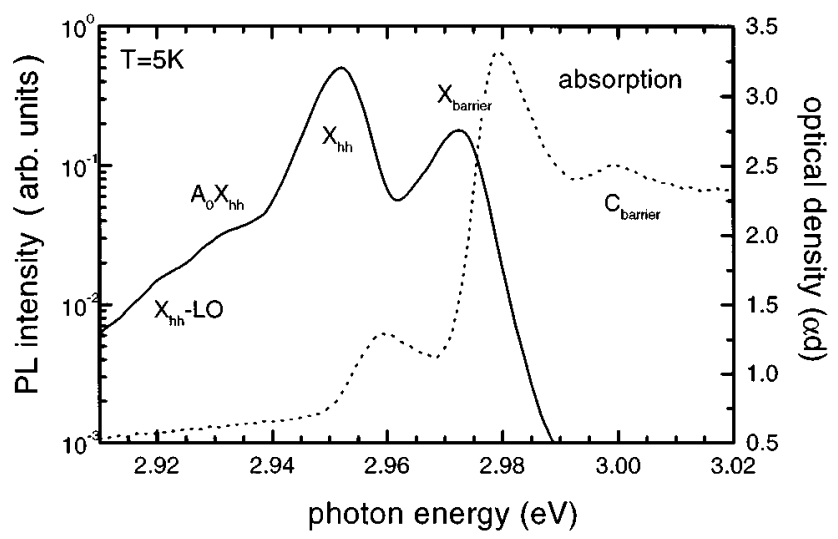

FIG. 1. PL under cw excitation at $3.44 \mathrm{eV}$ and $0.1 \mathrm{~W} / \mathrm{cm}^{2}$ and absorption of the $\mathrm{ZnSe} \mathrm{MQW}$ at $5 \mathrm{~K}$. The different transitions are labeled and explained in the text. 


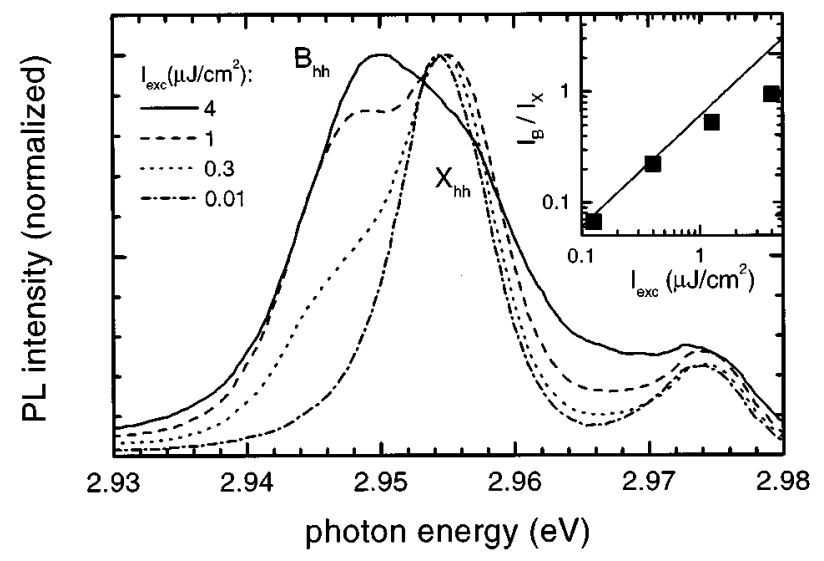

FIG. 2. PL spectra of the ZnSe MQW at $5 \mathrm{~K}$, and at a delay of $100 \mathrm{ps}$ after 1-ps excitation at $2.984 \mathrm{eV}$. The excitation intensities are given.

trally narrow 1-ps excitation pulses. The $100-\mathrm{fs}$ pulses were generated by frequency doubling of pulses from a self-modelocked Ti:sapphire laser pumped by an argon-ion laser. The narrow pulses were formed selecting a narrow spectral range from the broad pulses in a beam shaper, working as a subtractive double monochromator. The FWM signal was selected spatially by pinholes and detected spectrally resolved by a combination of a spectrometer and an optical multichannel analyzer.

To determine the biexciton binding-energy distribution, we use three-beam FWM with two coincident narrow pump pulses in the directions $\vec{k}_{1}$ and $\vec{k}_{2}$ and a broad probe pulse $\vec{k}_{3}$ delayed by the time $t_{13}$. All pulses are colinearly polarized, and the signal is detected in the direction $\vec{k}_{3}+\vec{k}_{2}-\vec{k}_{1}$ (backscattered). The observed biexciton and exciton signals decay in parallel as a function of delay $t_{13}$, showing an exciton dephasing time of 20 ps. The diffraction efficiency, e.g., the signal corrected by the probe spectrum, is shown in Fig. 3 for a delay $t_{13}$ of 2 ps. The small positive delay is chosen to minimize the effects of spectral diffusion. ${ }^{17}$ The spectrum shows the exciton and biexciton contributions, which have widths of 1.6 and $2.7 \mathrm{meV}$, respectively. This

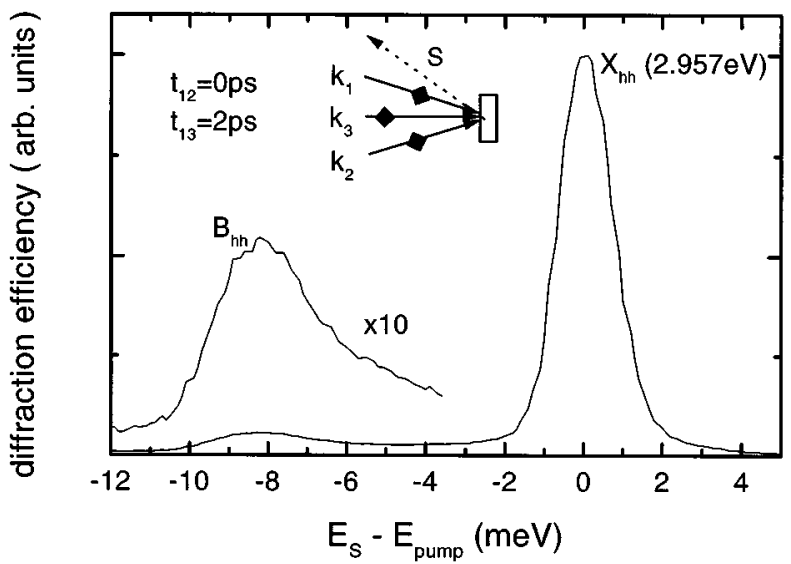

FIG. 3. Time-integrated, spectrally resolved, copolarized diffraction efficiency 2 ps after pumping the sample with two narrow pulses at $2.957 \mathrm{eV}$ of $1.8-\mathrm{meV}$ width. The diffraction geometry is shown in the inset. deconvolves to a biexciton binding energy distribution of $2.2-\mathrm{meV}$ full width at half maximum centered at $8.5 \mathrm{meV}$. The distribution is found to be nearly independent on the exciton localization energy $\left(E_{\text {pump }}=2.953-2.962 \mathrm{eV}\right)$.

The distribution of biexciton binding energies leads to a fast decay of the biexciton photon echo in two-beam FWM experiments, even in the absence of biexciton dephasing, as we show in the following. The spectrally resolved FWM signal in backward geometry in the direction $2 \vec{k}_{2}-\vec{k}_{1}$ is displayed in Fig. 4(a) as a function of delay time $t_{12}$ between the broad, colinearly polarized excitation pulses in the directions $\vec{k}_{1}$ and $\vec{k}_{2}$. The main signal is due to the photon echo of the excitons, showing a decay time of 5 ps. The corresponding exciton dephasing time of $20 \mathrm{ps}$ is comparable to values measured in $\mathrm{ZnSe} / \mathrm{Zn}_{x} \mathrm{Cd}_{1-x} \mathrm{Se}$ QW's. ${ }^{18}$ The biexciton signal, redshifted from the exciton signal, is present only for short delay times, and is also a photon echo. ${ }^{19}$ It shows a fast, stronger than exponential decay, with a mean decay time of about 400 fs. Between exciton and biexciton resonances, a strong oscillation is observed due to the excitonbiexciton quantum beat. The beat period of 480 fs corresponds to an energy splitting of $8.6 \mathrm{meV}$, in agreement with the spectrally measured biexciton binding energy of 8.5 $\mathrm{meV}$. The oscillations start with a minimum (maximum) at $t_{12}=0$ for copolarized (cross-polarized pulses), in agreement with theoretical predictions taking into account the two spin states of the excitons. ${ }^{9}$ For negative delay, the FWM signal decays very fast due to the dominating inhomogeneous broadening, in contrast to the biexcitonic quantum beats at negative delay times in homogeneously broadened systems. ${ }^{20,21}$

To separate the exciton and biexciton contributions, we use a spectrally narrow $(1.7 \mathrm{meV})$ pump pulse $\vec{k}_{1}$. The resulting FWM signal for copolarized pump and probe [Fig. 4(c)] shows a spectrally sharp response of the selected excitons at the pump energy, again revealing a decay time of 5 ps. Additionally, a low-energy sideband due to the biexciton photon echo is observed for short delays $t_{12}$. Its maximum is redshifted by the biexciton binding energy of $8.5 \mathrm{meV}$ from the exciton signal. The sideband remains for cross-polarized excitation, while the exciton signal is strongly suppressed, as expected for a biexciton photon echo. ${ }^{16,9}$

The fast, intensity-independent decay of the biexciton signal was observed previously in $\mathrm{CdS}_{x} \mathrm{Se}_{1-x}$ mixed crystals ${ }^{10}$ and GaAs QW's, ${ }^{19,9}$ and was interpreted as a fast dephasing of the biexciton state. However, a biexciton dephasing which is much faster than the exciton dephasing is inconsistent with the observed localized biexciton linewidth in GaAs QW's of $30 \mu \mathrm{eV}$, smaller than the exciton linewidth. ${ }^{3}$ The localized biexciton dephasing is also expected to be slower than the exciton dephasing due to the missing spin scattering of the biexcitons, present between the nearly degenerate exciton spin states. The exciton spin relaxation, which in GaAs QW's takes $20-50 \mathrm{ps},{ }^{22,23}$ probably determines the present exciton dephasing time of $20 \mathrm{ps}$. Consequently, the fast biexciton signal decay cannot be due to a microscopic dephasing. It is rather a consequence of the distribution of binding energies, giving rise to a destructive interference within the biexciton signal. This mechanism was previously predicted for the quantum beat in a $V$-type tree-level system such as 


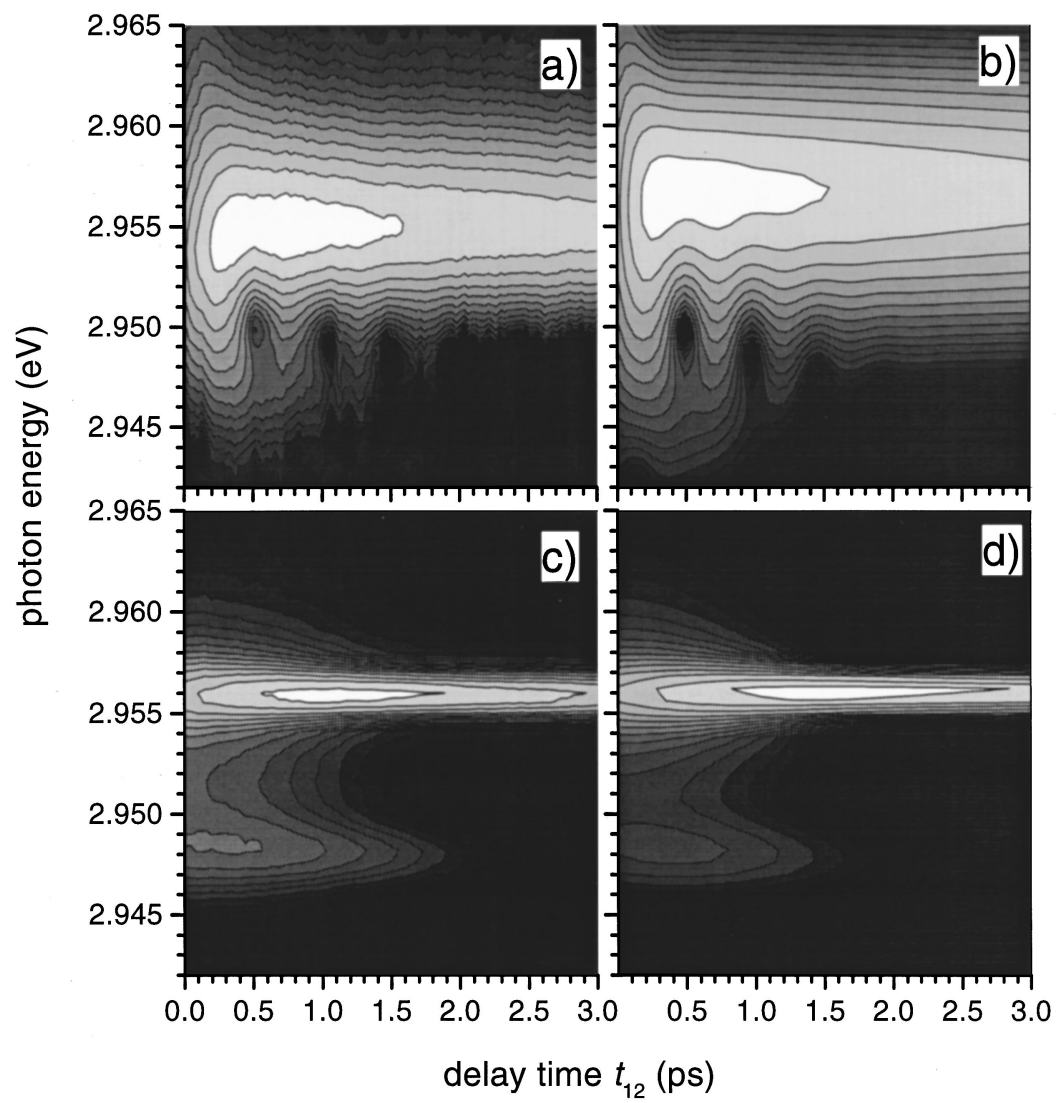

FIG. 4. Time-integrated, spectrally resolved, colinearly polarized FWM signal intensity in selfdiffraction and its theoretical simulation as a function of delay time. The contours are on a logarithmic scale covering three decades. (a) Experimental data using broad pump and probe pulses. (b) Simulation for (a) with Eq. (3) using $\sqrt{2} M_{B}=M_{X}, \Gamma_{B}=\Gamma_{X}=9 \mathrm{meV}, \gamma_{X}=0.033 \mathrm{meV}$, and $\gamma_{B}=0 \mathrm{meV}$, and $\lambda=0.97$. (c) Experimental data for narrow pump and broad probe pulses. The pump spectrum is centered at $2.9559 \mathrm{eV}$ and has a width of $1.7 \mathrm{meV}$. (d) Simulation for (c) with Eq. (3) using $\Gamma_{X}=1.7 \mathrm{meV}, \Gamma_{B}=2.8 \mathrm{meV}$, $\lambda=0.61$; other parameters are as in (b). the heavy-hole-light-hole exciton system. ${ }^{24,25}$ In the excitonbiexciton case, we have to consider an $E$-type level system, which is depicted schematically in Fig. 5. The interference of the third order polarizations $P_{X}^{(3)}$ and $P_{B}^{(3)}$, giving rise to the excitonic and biexcitonic photon echo, respectively, leads to the exciton-biexciton quantum beat oscillations in the FWM signal with delay time.

The fast decay of the biexciton signal $P_{B}^{(3)}$ can be explained as follows: For a certain signal photon energy, a range $\Delta E_{X}$ of exciton energies contributes, that is given by the distribution of biexcitonic binding energies. Consequently, the biexciton signal is subject to a destructive interference of the polarizations from the different exciton energies during the delay $t_{12}$ between the first and second pulses.
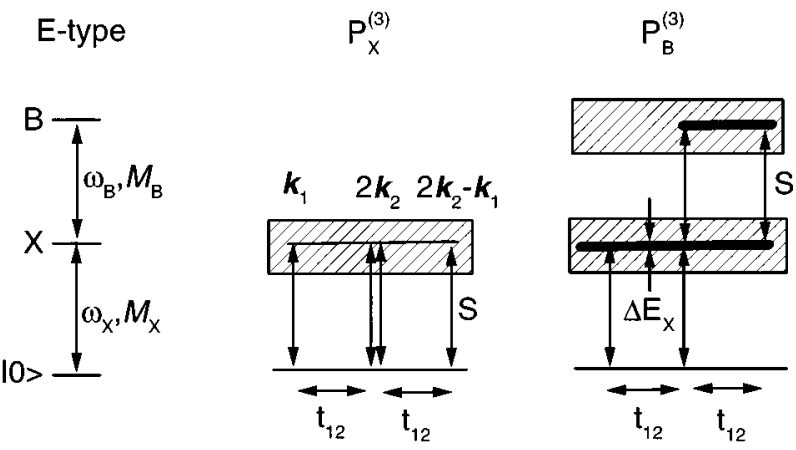

FIG. 5. Schematic representation of the level system under consideration, and of the mechanisms leading to the exciton photon echo signal $P_{X}^{(3)}$ and the biexciton photon echo signal $P_{B}^{(3)}$. The temporal sequence of the pump, probe, and signal $(S)$ pulses is indicated.
The biexciton signal decay is then determined by the Fourier transform of the distribution of biexciton binding energies.

To model the experiments quantitatively, taking into account the exciton and the biexciton transitions as well as their inhomogeneous broadening and the inhomogeneous width of the biexciton binding energy, we follow the analytical approach of Erland et al. ${ }^{24} \mathrm{We}$ use a Gaussian distribution function $g\left(\delta \omega_{X}, \delta \omega_{B}, \lambda\right)$ describing the inhomogeneous broadening $\Gamma_{X}$ and $\Gamma_{B}$ of the exciton $\left(\omega_{X}\right)$ and biexciton $\left(\omega_{B}\right)$ transition frequencies, with the correlation parameter $0 \leqslant \lambda \leqslant 1$, given by

$$
\begin{aligned}
g\left(\delta \omega_{X}, \delta \omega_{B}, \lambda\right)= & \frac{4 \ln (2)}{\pi \Gamma_{X} \Gamma_{B} \sqrt{1-\lambda^{2}}} \\
& \times \exp \left[\frac { - 4 \operatorname { l n } ( 2 ) } { 1 - \lambda ^ { 2 } } \left(\frac{\delta \omega_{X}^{2}}{\Gamma_{X}^{2}}-2 \lambda \frac{\delta \omega_{X} \delta \omega_{B}}{\Gamma_{X} \Gamma_{B}}\right.\right. \\
& \left.\left.+\frac{\delta \omega_{B}^{2}}{\Gamma_{B}^{2}}\right)\right]
\end{aligned}
$$

The spectrally resolved, time-integrated third-order polarization $P_{\tau \geqslant 0}^{(3)}(\tau, \omega)$ of a single localized exciton-biexciton system is calculated for infinitely short excitation pulses by a perturbative expansion of the solution of the optical Bloch equations for an $E$-type system (see Fig. 5) with only the ground state populated initially, ${ }^{26}$

$$
P_{\tau \geqslant 0}^{(3)}(\tau, \omega) \propto\left(\frac{2 M_{X}^{4}}{\omega_{X}-\omega}-\frac{M_{X}^{2} M_{B}^{2}}{\omega_{B}-\omega}\right) \exp \left[\left(i \omega_{X}-\gamma_{X}\right) \tau\right]
$$


Here $\gamma_{X}, \gamma_{B}$ and $M_{X}, M_{B}$ are the exciton and biexciton homogeneous linewidths and optical dipole matrix elements, respectively. We calculate the third-order polarization of the inhomogeneously broadened system $P_{\tau \geqslant 0}^{(3), \text { inh }}$ by summing $P_{\tau \geqslant 0}^{(3)}$ over the distribution $g\left(\delta \omega_{X}, \delta \omega_{B}, \lambda\right)$ of exciton energies $\omega_{X}+\delta \omega_{X}$ and biexciton energies $\omega_{B}+\delta \omega_{B}$ :

$$
\begin{aligned}
P_{\tau \geqslant 0}^{(3), \operatorname{inh}}(\tau, \omega) & \propto \frac{2 M_{X}^{4}}{\Gamma_{X}} \operatorname{erfc}\left[2 \sqrt{\ln 2}\left(\frac{\gamma_{X}+i\left(\omega_{X}-\omega\right)}{\Gamma_{X}}-\frac{\Gamma_{X} \tau}{8 \ln 2}\right)\right] \\
& \times \exp \left(\frac{4 \ln 2}{\Gamma_{X}^{2}}\left[\gamma_{X}+i\left(\omega_{X}-\omega\right)\right]^{2}+2\left(i \omega-\gamma_{X}\right) \tau\right) \\
& -\frac{M_{X}^{2} M_{B}^{2}}{\Gamma_{B}} \operatorname{erfc}\left[2 \sqrt{\ln 2}\left(\frac{\gamma_{B}+i\left(\omega_{B}-\omega\right)}{\Gamma_{B}}-\frac{\lambda \Gamma_{X} \tau}{8 \ln 2}\right)\right] \\
& \times \exp \left[\frac{4 \ln 2}{\Gamma_{B}^{2}}\left[\gamma_{B}+i\left(\omega_{B}-\omega\right)\right]^{2}-\frac{\left(1-\lambda^{2}\right) \Gamma_{X}^{2}}{16 \ln 2} \tau^{2}\right. \\
& \left.+\left(i\left(\omega_{X}+\omega\right)-\gamma_{X}-\lambda \frac{\Gamma_{X}}{\Gamma_{B}}\left[\gamma_{B}+i\left(\omega_{B}-\omega\right)\right]\right)\right] .
\end{aligned}
$$

The first term in Eq. (3) corresponds to the exciton signal $P_{X}^{(3)}$, the second term to the biexciton signal $P_{B}^{(3)}$. The main difference in their time dependence is the Gaussian decay of the biexciton contribution with the half time of $4 / \Gamma_{X} / \sqrt{\left(1-\lambda^{2}\right)}$. The origin of the fast biexciton signal decay is thus the inhomogeneous broadening of the biexciton binding energy, which is controlled here by the correlation $\lambda$.

The calculated FWM signal $\left|P_{\tau \geqslant 0}^{(3) \text {,inh }}\right|^{2}$, corresponding to the experimental conditions of the FWM data in Figs. 4(a) and 4(c), are given in Figs. 4(b) and 4(d). The biexciton dephasing is assumed to be absent in the calculations. The correlation $\lambda$ is chosen to reproduce the $2.2-\mathrm{meV}$ inhomogeneous width of the biexciton binding energy. All qualitative features are reproduced by the simulation, showing the validity of the applied model and of the given qualitative explanation. The experimental signal (a) is spectrally more narrow than the simulation (b) due to the finite spectral width of the exciting pulses. This also explains the lower intensity of the biexciton signal in the simulation (d) compared to the experiment.

In conclusion, we have determined the binding-energy distribution of localized biexcitons in a narrow ZnSe QW. It shows a width of $2.2 \mathrm{meV}$ centered at $8.5 \mathrm{meV}$. The decay of the biexcitonic signal in four-wave mixing is determined by this distribution rather than by a microscopic dephasing of the biexcitons, as is shown by an analytical model reproducing the experimental results. The localized biexciton dephasing time is expected to be longer than the exciton dephasing time and cannot be extracted from standard FWM experiments.

The authors want to thank D. Birkedal for helpful discussions. This work was supported by the Danish Ministries of Research and Industry in the framework of the Center for Nanostructures.
${ }^{1}$ Y. Z. Hu, S. W. Koch, M. Lindberg, N. Peyghambarian, E. L. Pollock, and F. F. Abraham, Phys. Rev. Lett. 64, 1805 (1990).

${ }^{2}$ K. I. Kang, A. D. Kepner, S. V. Gaponenko, S. W. Koch, Y. Z. $\mathrm{Hu}$, and N. Peyghambarian, Phys. Rev. B 48, 15449 (1993).

${ }^{3}$ K. Brunner, G. Abstreiter, G. Böhm, G. Tränkle, and G. Weimann, Phys. Rev. Lett. 73, 1138 (1994).

${ }^{4}$ J. Singh, D. Birkedal, V. G. Lyssenko, and J. M. Hvam, Phys. Rev. B 53, 15909 (1996).

${ }^{5}$ D. Birkedal, J. Singh, V. G. Lyssenko, J. Erland, and J. M. Hvam, Phys. Rev. Lett. 76, 672 (1996).

${ }^{6}$ J. R. Haynes, Phys. Rev. Lett. 17, 860 (1966).

${ }^{7}$ A. Ourmazd, D. W. Taylor, J. Cunningham, and C. W. Tu, Phys. Rev. Lett. 62, 933 (1989).

${ }^{8}$ D. Gammon, B. V. Shanabrook, and D. S. Katzer, Phys. Rev. Lett. 67, 1547 (1991).

${ }^{9}$ T. F. Albrecht, K. Bott, T. Meier, A. Schulze, M. Koch, S. T. Cundiff, J. Feldmann, W. Stolz, P. Thomas, S. W. Koch, and E. O. Göbel, Phys. Rev. B 54, 4436 (1996).

${ }^{10}$ J.-Y. Bigot, A. Daunois, J. Oberlé, and J.-C. Merle, Phys. Rev. Lett. 71, 1820 (1993)

${ }^{11}$ F. Yang, M. Wilkinson, E. J. Austin, and K. P. O’Donnell, Phys. Rev. Lett. 70, 323 (1993).

${ }^{12}$ Y. Yamada, T. Mishina, Y. Masumoto, Y. Kawakami, J. Suda, S. Fujita, and S. Fujita, Phys. Rev. B 52, R2289 (1995).

${ }^{13}$ F. Kreller, M. Lowisch, J. Puls, and F. Henneberger, Phys. Rev. Lett. 75, 2420 (1995).
${ }^{14}$ Q. Fu, D. Lee, A. Mysyrowicz, A. V. Nurmikko, R. L. Gunshor, and L. A. Kolodziejski, Phys. Rev. B 37, 8791 (1988).

${ }^{15}$ V. Kozlov, P. Kelkar, A. V. Nurmikko, C. C. Chu, D. C. Grillo, J. Han, C. G. Hua, and R. L. Gunshor, Phys. Rev. B 53, 10837 (1996).

${ }^{16}$ T. Häupl, H. Nickolaus, F. Henneberger, and A. Schülzgen, Phys. Status Solidi B 194, 219 (1996).

${ }^{17}$ H. Wang, M. Jiang, and D. G. Steel, Phys. Rev. Lett. 65, 1255 (1990).

${ }^{18}$ A. J. Fischer, D. S. Kim, J. Hays, W. Shan, J. J. Song, D. B. Eason, J. Ren, J. F. Schetzina, H. Luo, and J. K. Furdyna, Phys. Rev. B 50, 17643 (1994).

${ }^{19}$ T. Saiki, M. Kuwata-Gonokami, T. Matsusue, and H. Sakaki, Phys. Rev. B 49, 7817 (1994).

${ }^{20}$ E. J. Mayer, G. O. Smith, V. Heuckeroth, J. Kuhl, K. Bott, A. Schulze, T. Meier, D. Bennhardt, S. W. Koch, P. Thomas, R. Hey, and K. Ploog, Phys. Rev. B 50, 14730 (1994).

${ }^{21}$ B. F. Feuerbacher, J. Kuhl, and K. Ploog, Phys. Rev. B 43, 2439 (1991).

${ }^{22}$ S. Bar-Ad and I. Bar-Joseph, Phys. Rev. Lett. 68, 349 (1992).

${ }^{23}$ R. E. Worsley, N. J. Traynor, T. Grevatt, and R. T. Harley, Phys. Rev. Lett. 76, 3224 (1996).

${ }^{24}$ J. Erland, K.-H. Pantke, V. Mizeikis, V. G. Lyssenko, and J. M. Hvam, Phys. Rev. B 50, 15047 (1994).

${ }^{25}$ S. T. Cundiff, Phys. Rev. A 49, 3114 (1994).

${ }^{26}$ J. Erland and I. Balslev, Phys. Rev. A 48, R1765 (1993). 